\title{
Heterogeneity in the colorectal primary tumor and the synchronous resected liver metastases prior to and after treatment with an anti-EGFR monoclonal antibody
}

\author{
DANIELA ADUA ${ }^{1,2}$, FRANCESCA DI FABIO ${ }^{2}$, GIORGIO ERCOLANI ${ }^{3}$, MICHELANGELO FIORENTINO $^{4}$, \\ ELISA GRUPPIONI $^{4}$, ANNALISA ALTIMARI ${ }^{4}$, FABIOLA LORENA ROJAS LIMPE ${ }^{2}$, \\ NICOLA NORMANNO $^{5,6}$, ANTONIO DANIELE PINNA ${ }^{3}$ and CARMINE PINTO $^{7}$
}

\begin{abstract}
${ }^{1}$ Department of Experimental, Diagnostic and Specialty Medicine, University of Bologna, I-40126 Bologna; ${ }^{2}$ Medical Oncology Unit, Sant'Orsola-Malpighi Hospital; ${ }^{3}$ Department of General Surgery and Transplantation, Sant'Orsola-Malpighi Hospital, University of Bologna; ${ }^{4}$ Pathology Unit, Addarii Institute, Sant'Orsola-Malpighi Hospital, University of Bologna, I-40138 Bologna; ${ }^{5}$ Cell Biology and Biotherapy Unit; ${ }^{6}$ Laboratory of Pharmacogenomics, Centro di Ricerche Oncologiche di Mercogliano (CROM), Istituto Nazionale Tumori ‘Fondazione Giovanni Pascale' IRCCS, I-80131 Naples; ${ }^{7}$ Medical Oncology

Unit, Clinical Cancer Centre, IRCCS-Arcispedale S. Maria Nuova, I-42123 Reggio Emilia, Italy
\end{abstract}

Received November 9, 2016; Accepted March 9, 2017

DOI: $10.3892 /$ mco.2017.1270

\begin{abstract}
Molecular heterogeneity between primary tumors (PTs) and synchronous resected liver metastasis in colorectal cancer (CRC) has potential relevance in treatment strategies. Next-generation sequencing (NGS) may be able to increase the chances of identifying multiple molecular driver alterations, calling for therapy. The aim of the present study was to evaluate mutations in PT and synchronous resected liver metastases for patients with Kirsten rat sarcoma 2 viral oncogene homolog (KRAS) exon 2 wild-type metastatic (m)CRC who underwent chemotherapy (CT) featuring an anti-epidermal growth factor receptor (EGFR) monoclonal antibody. Genomic analysis was performed on 54 lesions from 7 patients with mCRC. For each patient, a PT biopsy or a surgical specimen was obtained prior to $\mathrm{CT}$, and the PT and all liver metastases resected following CT were analyzed. DNA libraries were generated using the Ion AmpliSeq Colon and Lung Cancer Panel, assessing the most frequent somatic mutations in 22 genes involved in colon tumorigenesis, and sequencing was performed on an Ion Personal Genome Machine system. A partial response was achieved in all the patients, with a median progression free survival time of 11 months (range, 3-21 months). All the patients were subjected to surgical liver metastasis resection. The median overall survival time was 31 months (range,
\end{abstract}

Correspondence to: Dr Daniela Adua, Department of Experimental, Diagnostic and Specialty Medicine, University of Bologna, Via P. Albertoni 15, I-40126 Bologna, Italy

E-mail: aduadani@hotmail.com

Key words: intratumor heterogeneity, metastatic colorectal cancer, liver metastasis, next-generation sequencing, cetuximab
4-46 months). Molecular analysis of the genes correlated with the target therapy, suggesting significant intratumor heterogeneity, as revealed by the different mutational landscape of certain PTs and synchronous resected liver metastases following systemic therapy when compared with the PT prior to treatment. In particular, the loss and acquisition of mutations in KRAS, neuroblastoma RAS viral oncogene homolog (NRAS), tumor protein p53 (TP53), the p110 $\alpha$ catalytic subunit of phosphoinositide 3-kinase (PIK3CA), F-box/WD repeat-containing protein 7 (FBXW7) and phosphatase and tensin homolog (PTEN) were observed. In addition, one patient developed a mucinous pattern following systemic CT. Taken together, the results of the present study demonstrated that intratumor heterogeneity is likely to affect the response to therapy, and to drive acquired resistance to targeted agents. The preliminary data also suggest a potential role for NGS in the evaluation of biological drug resistance, affecting future sequential treatment strategies.

\section{Introduction}

Colorectal cancer (CRC) is the third most common cancer in Western countries in terms of incidence and mortality. With a trend of increasing incidence, it constitutes $8 \%$ of all new diagnoses of cancer in adulthood in Europe, placing itself in third place for men and second place for women (1). The disease is diagnosed in $80 \%$ of cases at an early stage, susceptible to curative surgery and possible adjuvant chemotherapy (CT), with a 5 -year recurrence rate that exceeds $35 \%$. In the remaining $20 \%$ of cases, the disease is diagnosed at an advanced stage [metastatic CRC (mCRC)], with a trend in overall survival (OS) that is closely dependent on the molecular characterization and the resection of metastatic sites (1).

Over the last 15 years, mCRC has been associated with an increase in survival rates, reaching an average of 31 months 
courtesy of the introduction of doublets/triplets of CT in combination with monoclonal antibodies (mAbs) directed against the vascular endothelial growth factor (VEGF) and the epidermal growth factor receptor (EGFR). Although biomarkers to select patients responding to anti-VEGF mAbs are not yet available, mutations in exons 2, 3 and 4 of the Kirsten rat sarcoma 2 viral oncogene homolog (KRAS) and neuroblastoma RAS viral oncogene homolog (NRAS) genes are predictive of resistance to anti-EGFR mAbs, whereas the Val600èGln (V600E) mutation of the B-Raf proto-oncogene (BRAF) has a strong prognostic role, predicting resistance to standard CT (2-6).

Therefore, mutations in the RAS and BRAF genes may identify subgroups of tumors with specific biological, pathological and clinical features, a phenomenon referred to as 'inter-tumor heterogeneity'. However, CRC is a complex disease, characterized by a number of genetic alterations that may also co-exist in the same tumor. In particular, it has been suggested that CRC is likely to be formed by different clones of cancer cells with distinct genotypic profiles. This intratumor heterogeneity is likely to markedly affect the progression of the disease, as well as the sensitivity and resistance to therapies. Notably, recent studies have also suggested that treatment of CRC patients with anti-EGFR agents produces an increase in intratumor heterogeneity, leading to the emergence of clones with different genetic alterations (7).

An additional important consequence of intratumor heterogeneity is the possible difference in genetic alterations between primary tumors (PTs) and metastases (8). Although a mutational concordance of KRAS, NRAS and BRAF between the PT and metastases in excess of $95 \%$ has been highlighted in different cases, this agreement begins to falter with the introduction of high-throughput genotyping techniques, such as targeted resequencing applications of next-generation sequencing (NGS) (9-11).

Focusing on the key role of the heterogeneity on the development and spread of the tumor, the authors of the present study speculated on whether the PT tissue of CRC should continue to offer a trustworthy overview of metastatic tissue, how the de novo resistance may impact on treatments with anti-EGFR mAbs, and the nature of the role of the secondary resistance on the progress of the disease.

Recent data have suggested that acquired resistance to anti-EGFR mAbs is driven by a number of molecular alterations, including mutations in KRAS, NRAS, BRAF, and other driver genes $(12,13)$. Furthermore, the higher sensitivity of NGS may permit the identification of mutations in RAS not identified by the standard Sanger sequencing technique, as highlighted by the analysis conducted in a subpopulation of the CAPRI-GOIM multicenter study $(13,14)$. In this regard, it was also suggested that low levels of KRAS mutations could justify an intrinsic resistance mechanism to anti-EGFR mAbs $(15,16)$.

In the present study, the genetic profile of the colorectal PT prior to and after CT, and of resected liver metastases removed post-CT in association with cetuximab, was assessed in order to investigate the genetic heterogeneity and the intrinsic and acquired resistance mechanisms in patients with mCRC.

\section{Patients and methods}

Study design and patient population. The working hypothesis adopted for the present study was to investigate the impact of intra- and inter-tumoral molecular heterogeneity between colorectal PTs and metastatic sites prior to and after treatment with cetuximab, in combination with doublet (folinic acid, fluorouracil and irinotecan, or FOLFIRI) or triplet (folinic acid, 5-fluorouracil, oxaliplatin and irinotecan, or FOLFOXIRI) CT in KRAS exon 2 wild-type chemo-naive patients with synchronous potentially resectable liver metastases.

Seven cases of patients with wild-type KRAS exon 2 mCRC were evaluated at the Oncology Medical Unit of St. Orsola-Malpighi Hospital, Bologna, Italy, between June 2010 and February 2014 for a total of 54 analyzed lesions. The selected time period justifies the population selected only for the absence of mutations in KRAS exon 2.

All the patients provided their informed consent for the treatment of their genetic material for research purposes, and the present study was approved by the Ethical Committee of the S. Orsola-Malpighi Hospital.

Two patients (patient nos. 2 and 5) had undergone two-stage hepatectomy surgery interspersed with CT in combination with cetuximab, whereas others underwent surgery only after conversion treatment. The average number of treatment cycles carried out prior to the surgical resection was agreed for each individual case during the multidisciplinary meeting of the study, and for the treatment of liver metastases prior to clinical-instrumental re-evaluation, and was set equal to seven (range, 6-8 cycles).

Gene mutation analysis by NGS. NGS analysis was performed using genomic DNA extracted either from PT tissue obtained prior to systemic treatment or on liver metastases following either liver resection or biopsy. Formalin-fixed, paraffin-embedded (FFPE), circled tumor-rich (>70\%) areas (10- $\mu \mathrm{m}$ thick) were scraped off the slides using a sterile scalpel by manual microdissection, deparaffinized in xylene, and DNA was isolated using the GeneRead DNA FFPE kit (Qiagen GmbH, Hilden, Germany), according to the manufacturer's protocol. DNA quantification was performed using a Quantifiler ${ }^{\circledR}$ Human DNA Quantification kit (Thermo Fisher Scientific, Inc., Waltham, MA, USA). NGS was performed using the Ion System Personal Genome Machine (PGM) system (Life Technologies; ThermoFisher Scientific, Inc.), with all reagents supplied by Thermo Fisher Scientific, Inc. An amplicon library was produced from $10 \mathrm{ng}$ DNA from each sample using the Ion AmpliSeq ${ }^{\mathrm{TM}}$ Colon and Lung Research panel v2 (Thermo Fisher Scientific, Inc.), which generates 92 amplicons for analyses of 'hotspot' and targeted regions of 22 genes implicated in colon and lung cancers [namely, KRAS, EGFR, BRAF, p $110 \alpha$ catalytic subunit of phosphoinositide 3-kinase (PIK3CA), serine/threonine kinase 1 (AKT1), erb-b2 receptor tyrosine kinase (ERBB)2, phosphatase and tensin homolog (PTEN), NRAS, serine/threonine kinase 11 (STK11), mitogen-activated protein kinase kinase 1 (MAP2K1), anaplastic lymphoma kinase (ALK), discoidin domain-containing receptor 2 (DDR2), catenin $\beta 1$ (CTNNB1), MET proto-oncogene, tumor protein p53 (TP53), SMAD4, F-box/WD repeat-containing protein 7 (FBXW7), fibroblast 
Table I. Disease progression and mutation percentages, comparing PTs with srLm.

\begin{tabular}{|c|c|c|c|c|c|c|c|}
\hline $\begin{array}{l}\text { Patient } \\
\text { no. }\end{array}$ & $\begin{array}{c}\text { PFS } \\
\text { (months) }\end{array}$ & $\begin{array}{c}\text { OS } \\
\text { (months) }\end{array}$ & $\begin{array}{l}\text { Number of CT } \\
\text { lines subsequent } \\
\text { to the first }\end{array}$ & $\begin{array}{l}\text { Number of liver } \\
\text { resections after } \\
\text { the first }\end{array}$ & $\begin{array}{c}\text { PT }(\%) \\
\text { mutated genes } \\
\text { prior to CT }\end{array}$ & $\begin{array}{c}\text { PT }(\%) \\
\text { mutated genes } \\
\text { after CT }\end{array}$ & $\begin{array}{c}\text { srLm }(\%) \\
\text { mutated } \\
\text { genes after CT }\end{array}$ \\
\hline 1 & 14 & 46 & 4 & 1 & 9 & 14 & 9 \\
\hline 2 & 3 & 4 & 0 & 1 & 9 & - & 9 \\
\hline 3 & 9 & 40 & 1 & 1 & 0 & 0 & 0 \\
\hline 4 & 14 & 36 & 2 & 0 & 4 & - & 4 \\
\hline 5 & 9 & 21 & 2 & 1 & 4 & - & 9 \\
\hline 6 & 21 & 37 & 2 & 1 & 4 & - & 4 \\
\hline 7 & 8 & 12 & 2 & 0 & 14 & 14 & 14 \\
\hline
\end{tabular}

PT, primary tumor; srLm, synchronous resected liver metastasis; PFS, progression free survival; OS, overall survival; CT, chemotherapy.

growth factor receptor (FGFR)3, NOTCH1, ERBB4, FGFR1 and FGFR2].

The manufacturer's protocols were followed without modification. Briefly, after amplification of the target sequences, primer digestion was performed, and barcode adapters (Ion Xpress Barcode Adapters; Thermo Fisher Scientific, Inc.) were ligated to the amplicons. Amplicons were purified using Agencourt AMPure XP (Beckman Coulter, Brea, CA, USA). Library quantification was subsequently performed using the Ion Library TaqMan ${ }^{\mathrm{TM}}$ Quantitation kit (Thermo Fisher Scientific, Inc.). The library was diluted in nuclease-free water to obtain a final concentration of $8 \mathrm{pM}$. Emulsion polymerase chain reaction (PCR) was performed using an Ion $\mathrm{PGM}^{\mathrm{TM}}$ Template OT2 200 kit on the Ion OneTouch ${ }^{\mathrm{TM}} 2$ instrument (Thermo Fisher Scientific, Inc.). The quality of the DNA following PCR was measured using the Qubit Ion Sphere ${ }^{\mathrm{TM}}$ Quality Control kit (Thermo Fisher Scientific, Inc.). Selective ion spheres with DNA were isolated (Ion PGM ${ }^{\mathrm{TM}}$ Enrichment Beads on an Ion OneTouch ${ }^{\mathrm{TM}}$ ES instrument), and sequenced on an Ion $316^{\mathrm{TM}}$ Chip kit v2 (5 samples/chip) or an Ion $318^{\mathrm{TM}}$ Chip kit v2 (10 samples/chip) using the Ion PGM $^{\text {TM }}$ Sequencing 200 kit v2 (Thermo Fisher Scientific, Inc.). Successful sequencing of a sample required at least 500,000 reads with a quality score $\geq$ Q20.

As tumor specimens were admixed with normal tissue, a minimum coverage of $500 \mathrm{X}$ with at least $10 \%$ frequency was used as cutoff for a variant to be considered true.

Sequence alignment and base calling was performed using Torrent Suite software v.4.4.3 (Thermo Fisher Scientific, Inc.), with Human Genome Build 19 (hg19) as the reference. Variant calling was performed with the Variant Caller v.4.4.3.3 plug-in using default 'Somatic-Low Stringency' settings. Variants were further filtered using Ion Reporter software v.4.4 (Thermo Fisher Scientific, Inc.) to meet the following criteria: Non-synonymous coding, an allele frequency $\geq 10 \%$, a total amplicon coverage of $\geq 500$, each variant coverage $>20$, a Phred-based quality score of $\geq 30$, and $\mathrm{P}<0.001$. This software also included the ClinVar, dpSNP (National Center for Biotechnology Information, Bethesda, MD, USA) and COSMIC (Wellcome Trust Sanger Institute, Cambridge, UK) databases, and, for missense mutations of unknown significance, in silico prediction tools, including SIFT (Sorting Intolerant From

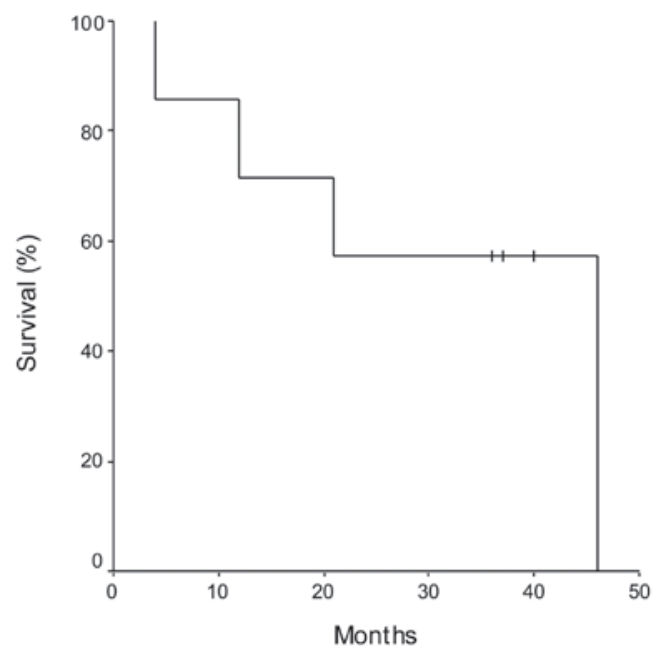

Figure 1. Kaplan-Meier analysis, depicting the overall survival curves.

Tolerant), PolyPhen (Polymorphism Phenotyping), PhyloP and Grantham.

The Integrative Genomics Viewer (IGV; Broad Institute, Cambridge, MA, USA) was used to visualize variants. Additionally, several of the detected missense mutations were confirmed using Sanger's sequencing.

\section{Results}

After the initial treatment, an objective partial response was achieved in all 7 patients (according to RECIST Criteria v.1.1), which allowed the previously planned surgical resection to be performed for all our patients.

Following the resection of the liver metastases, patients had a liver recurrence rate of $71 \%$; two patients $(28 \%$; patient nos. 3 and 6) were subjected to further liver surgery, with an expected benefit in terms of progression free survival (PFS; 25 and 9 months, respectively) and OS (40 and 37 months, respectively). Three out of seven patients (42\%) were subjected to a second line of treatment, containing an anti-VEGF antibody; two out of seven $(28 \%)$ patients were subjected to a third line of treatment, and $14 \%$ (one patient) was subjected to successive lines of treatment. 


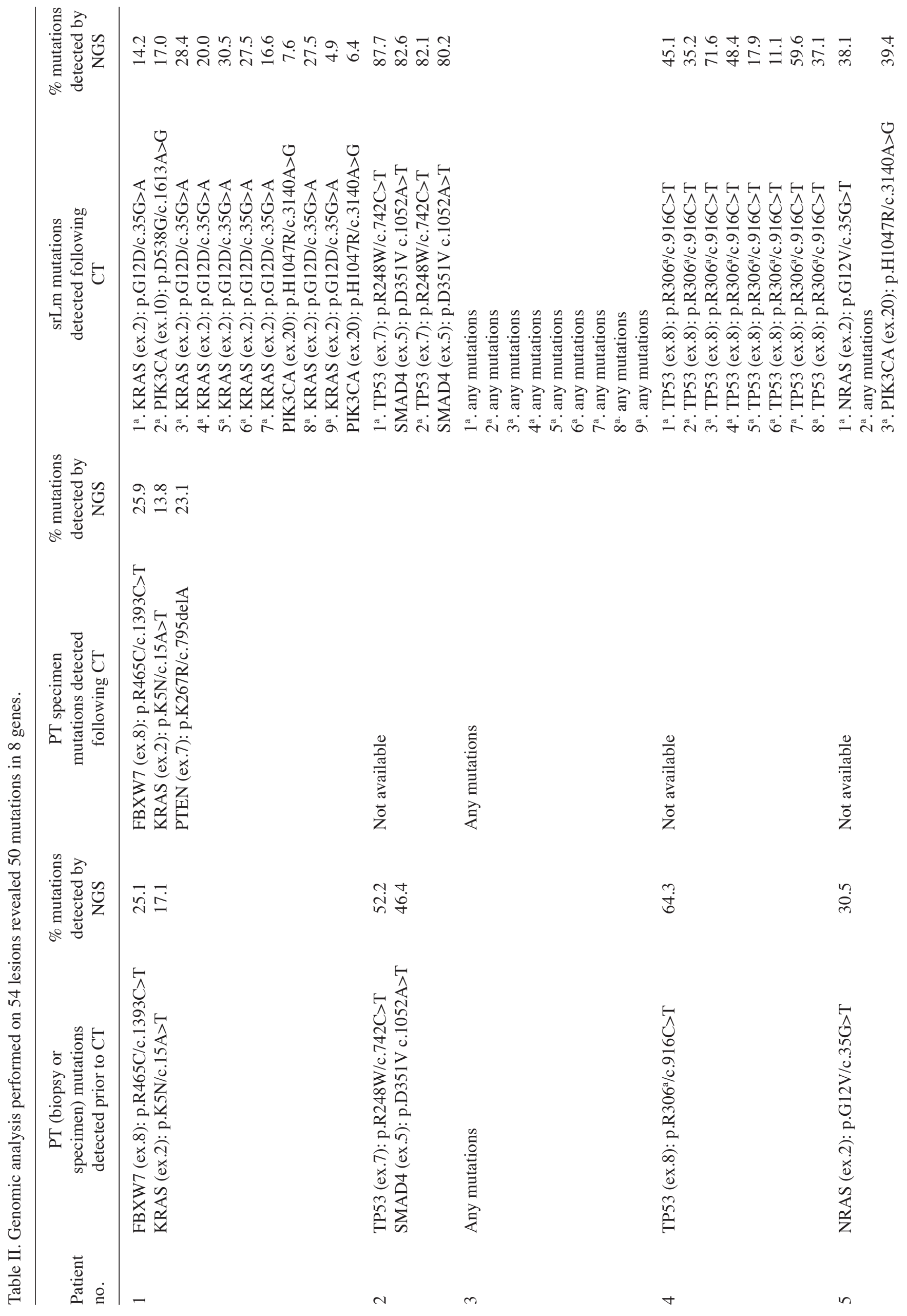




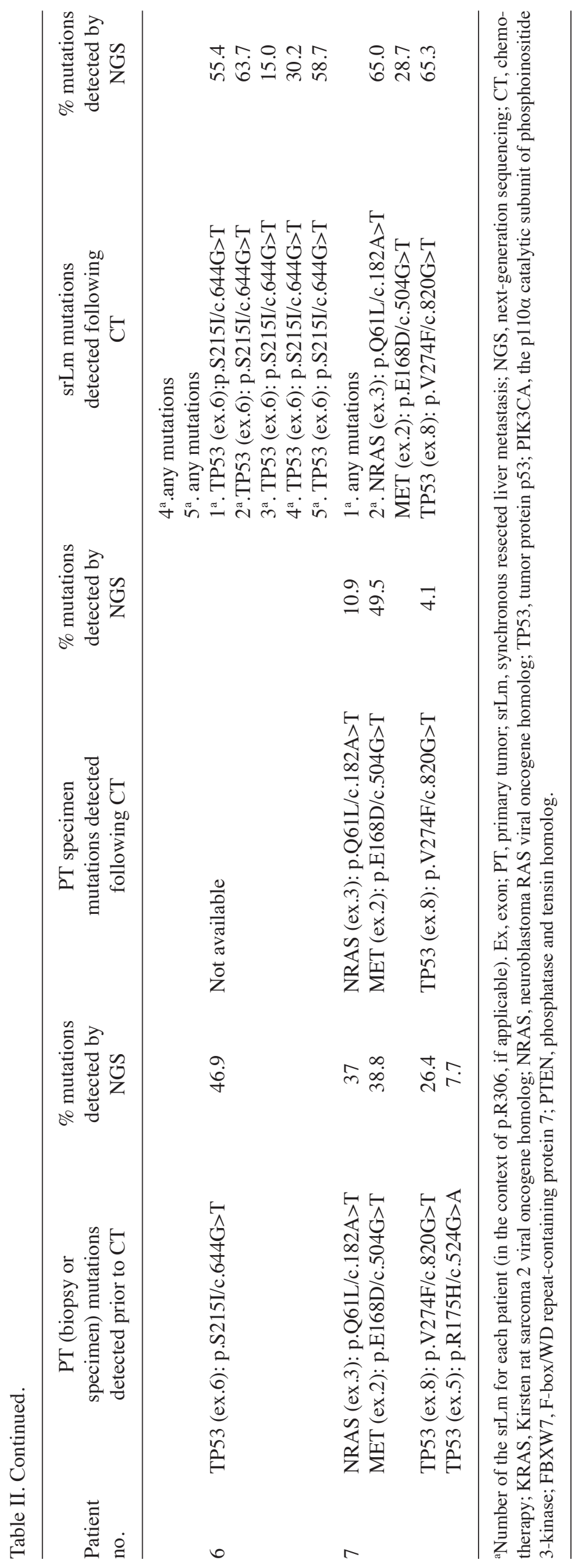




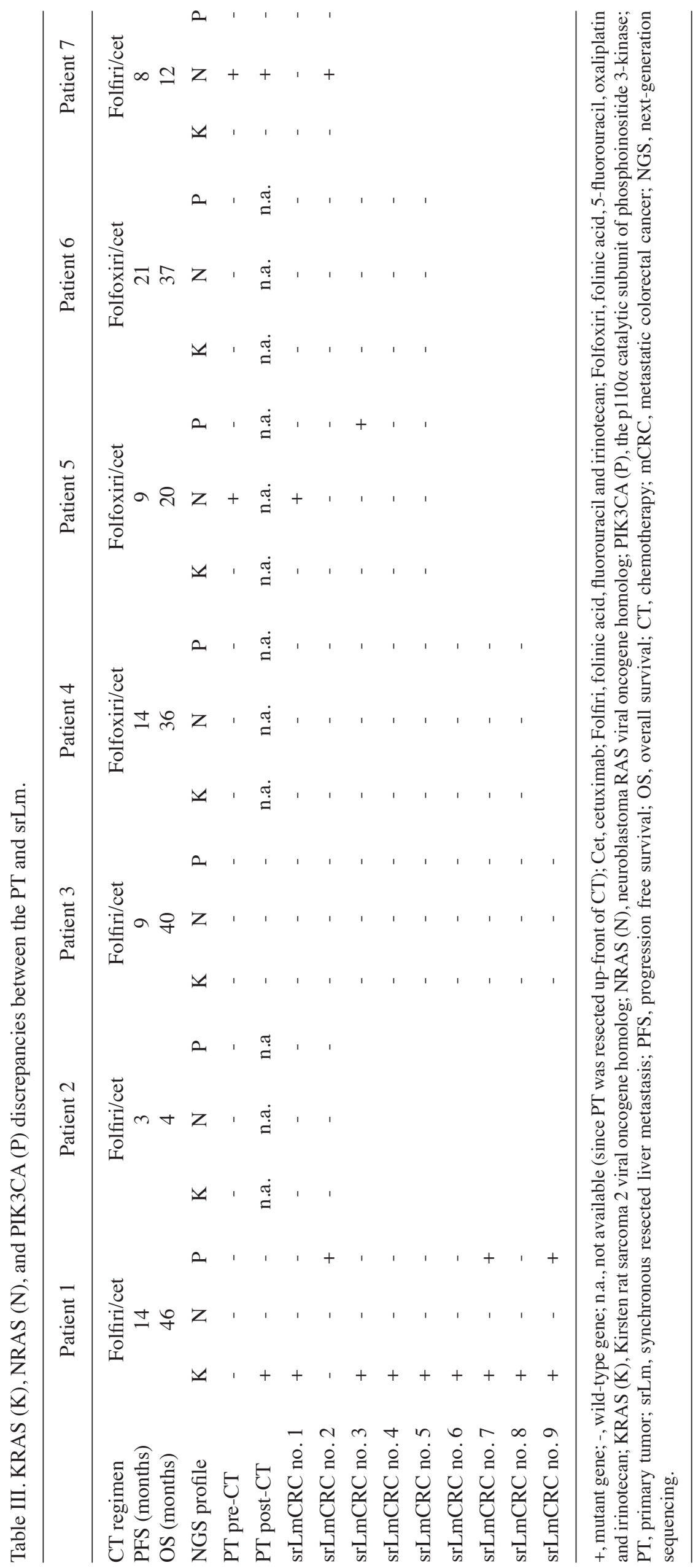


Using Kaplan-Meier analysis, the PFS, calculated as the time from liver resection and the disease progression, was 11 months (range, 3-21 months), whereas the OS was 31 months (range, 4-46 months) (Fig. 1). Patients who demonstrated a significant advantage in OS were those subjected to further liver surgery (patient nos. 3 and 6, equal to 40 and 37 months, respectively), and to subsequent lines of $\mathrm{CT}$, confirming the importance of a multidisciplinary approach to the treatment of mCRC (Table I).

The NGS analysis of pre- and post-treatment available tissues revealed 50 mutations in 8 genes: KRAS $(22 \%)$, NRAS (8\%), PIK3CA (8\%), MET (4\%), FBXW7 (6\%), PTEN (2\%), SMAD4 (8\%) and TP53 (42\%) (Table II).

Five of the 7 treated patients were of the RAS wild-type. Two had NRAS mutations that were retrospectively assessed, since the label of the drug allowed treatment of patients with KRAS exon 2 wild-type tumor at the time of the treatment. Mutations in TP53, SMAD4, FBXW7 and MET were also present in the PT from four of the seven patients prior to treatment.

As shown in Table III, the treatment produced marked changes in the mutational profile in 3 of the 7 patients, namely patient nos. 1, 5, and 7 .

Patient no.1 demonstrated genetic heterogeneity of the PT prior to and after $\mathrm{CT}$, with the appearance following CT plus cetuximab of exon 2 KRAS (p.K5N/c.15A >T) and exon 7 PTEN (p.K267R/c.795delA) mutations, and a marked increase in a mucinous pattern. In addition, the exon 2 KRAS mutation was identified in 7 of 9 resected liver metastases. In 3 of 9 metastases, different PIK3CA mutations were also detected (exon 10: p.D538 G/c.1613A>G; exon 20: p.H1047R/c.3140A>G). Notably, the exon 8 FBXW7 (p.R465C/c.1393C>T) mutation present in the PT prior to and after $\mathrm{CT}$ was not detected in the liver metastases.

Patient no. 5 had a mutation in NRAS exon 2 (p.G12 $\mathrm{V} / \mathrm{c} .35 \mathrm{G}>\mathrm{T}$ ) in the PT prior to and after treatment and in two of the five resected metastases, whereas the exon 20 PIK3CA variant (p.H1047R/c.3140A >G) was observed in one single metastasis.

Patient no. 7 had mutations in NRAS exon 3 (p.Q61L/ c.182A>T), TP53 exon 8 (p.V274F/c.820G >T) and MET exon 2 (p.E168D/c.504G $>$ T) in the PT prior to and after treatment, whereas the identical mutations were present in only one of the two resected liver metastases. An additional TP53 exon 5 (p.R175H/c.524G>A) mutation was observed only in the PT prior to $\mathrm{CT}$.

\section{Discussion}

NGS is a powerful technique that allows the study of multiple biomarkers in a single analysis. By applying this technique to tissue specimens obtained prior and after $\mathrm{CT}$, it has been possible to follow the molecular evolution of the disease in a small cohort of patients.

In agreement with previous studies, significant changes in KRAS mutation status were identified in one patient (patient no. 1). Indeed, several reports have revealed that RAS wild-type patients might develop RAS mutations following exposure to anti-EGFR mAbs (15). However, in the present study, loss of the FBXW7 mutation and the gain of a PIK3CA mutation in liver metastases were also identified. Changes in PIK3CA mutation status during treatment with anti-EGFR drugs have previously been described, although a clear correlation with resistance to anti-EGFR mAbs was not identified (17). Mutations in FBXW7 have been recently reported as potential markers of resistance to anti-EGFR mAbs (18). However, the disappearance of the FBXW7 mutation from cells following response to cetuximab-based therapy argues against this hypothesis. Such differences could also be due to a relatively lower ability of cells with the FBXW7 mutation to establish distant metastases in the liver. Another noteworthy observation is the switch to a mucinous pattern that was not observed prior to therapy. Such a histological change may represent a novel pathway of resistance to anti-EGFR mAbs.

In two distinct patients, NRAS mutations that were evident prior to treatment were not detected in all, or in selected, liver metastases. Again, the lack of liver tissue prior to treatment in the present study prevents the conclusion from being drawn that changes in the mutational pattern have occurred after therapy, rather than representing a different ability of tumor clones to establish liver metastases. Nevertheless, these findings highlight how the dynamics of clonal evolution between the PT and distant localizations of the disease are highly complex.

No mutations of the extracellular domain of the EGFR were identified in this small cohort of patients after exposure to cetuximab. Different studies have suggested that development of these mutations is one of the main mechanisms of acquired, but not intrinsic, resistance to cetuximab (19-21). It must be emphasized that the majority of the available data have been obtained with liquid biopsy, which still requires further validation (22).

In conclusion, the present study has highlighted marked differences between pre- and post-treatment biopsies from patients with CRC. These changes are likely to be due to the selection of sub-clones by therapy, thus suggesting a high level of intratumor heterogeneity that could markedly affect the response to therapy.

\section{References}

1. Arnold M, Sierra MS, Laversanne M, Soerjomataram I, Jemal A and Bray F: Global patterns and trends in colorectal cancer incidence and mortality. Gut 66: 683-691, 2017.

2. Prenen H, Tejpar S and Van Cutsem E: Impact of molecular markers on treatment selection in advanced colorectal cancer. Eur J Cancer 45 (suppl 1): S70-S78, 2009.

3. Bokemeyer C, Van Cutsem E, Rougier P, Ciardiello F, Heeger S, Schlichting M, Celik I and Köhne CH: Addition of cetuximab to chemotherapy as first-line treatment for KRAS wild-type metastatic colorectal cancer: Pooled analysis of the CRYSTAL and OPUS randomised clinical trials. Eur J Cancer 48: 1466-1475, 2012.

4. Douillard JY, Oliner KS, Siena S, Tabernero J, Burkes R, Barugel M, Humblet Y, Bodoky G, Cunningham D, Jassem J, et al: Panitumumab-FOLFOX4 treatment and RAS mutations in colorectal cancer. N Engl J Med 369: 1023-1034, 2013.

5. Stintzing S, Jung A, Rosssius L and Heinemann V: Analysis of KRAS/NRAS and BRAF mutations in FIRE-3: A randomized phase III study of Folfiri plus cetuximab or bevacizumab as first-line treatment for wild-type (WT) KRAS (exon 2) metastatic colorectal cancer (mCRC) patients. Eur J Cancer 49 (suppl 3): Abstract 17, 2013.

6. Schwartzberg LS, Rivera F, Karthaus M, Fasola G, Canon JL, $\mathrm{Yu} \mathrm{H}$, Oliner KS and Go WY: Analysis of KRAS/NRAS mutations in PEAK: A randomized phase II study of FOLFOX6 plus panitumumab (pmab) or bevacizumab (bev) as first-line treatment (tx) for wild-type (WT) KRAS (exon 2) metastatic colorectal cancer (mCRC). J Clin Oncol; 13 (suppl 3): abstr 3631, 2013. 
7. De Roock W, De Vriendt V, Normanno N, Ciardiello F and Tejpar S: KRAS, BRAF, PIK3CA, and PTEN mutations: Implications for targeted therapies in metastatic colorectal cancer. Lancet Oncol 12: 594-603, 2011.

8. Gerlinger M, Rowan AJ, Horswell S, Larkin J, Endesfelder D, Gronroos E, Martinez P, Matthews N, Stewart A, Tarpey P, et al: Intratumor heterogeneity and branched evolution revealed by multiregion sequencing. N Engl J Med 366: 883-892, 2012.

9. Kopetz S, Overman MJ, Chen K, Lucio-Eterovic AK, Kee BK, Fogelman DR, Dasari A, Raghav KPS, Sanchez EV, Phillips J, et al: Mutation and copy number discordance in primary versus metastatic colorectal cancer (mCRC). J Clin Oncol 32 (Suppl: abstr 3509): 5S, 2014

10. Adua D, Altimari A, Gruppioni E, G Ercolani, Llimpe FLR, Fabio FD, Fiorentino $M$ and Pinna AD, Pinto C; Medical Oncology Unit, S. Orsola-Malpighi Hospital, Bologna, Italy et al: Molecular evaluation of primary tumor (PT) and synchronous liver metastasis in colorectal cancer (srLmCRC) patients after cetuximab-based chemotherapy. J Clin Oncol 32 (Suppl; abstr 3624): 5S, 2014

11. Mao C, Wu XY, Yang ZY, Threapleton DE, Yuan JQ, Yu YY and Tang JL: Concordant analysis of KRAS, BRAF, PIK3CA mutations, and PTEN expression between primary colorectal cancer and matched metastases. Sci Rep 5: 8065, 2015.

12. Sartore-Bianchi A, Di Nicolantonio F, Nichelatti M, Molinari F, De Dosso S, Saletti P, Martini M, Cipani T, Marrapese G, Mazzucchelli L, et al: Multi-determinants analysis of molecular alterations for predicting clinical benefit to EGFR-targeted monoclonal antibodies in colorectal cancer. PLoS One 4: e7287, 2009.

13. NormannoN, Rachiglio AM,Lambiase M, Martinelli E,Fenizia F, Esposito C, Roma C, Troiani T, Rizzi D, Tatangelo F, et al: Heterogeneity of KRAS, NRAS, BRAF and PIK3CA mutations in metastatic colorectal cancer and potential effects on therapy in the CAPRI GOIM trial. Ann Oncol 26: 1710-174, 2015.

14. Ciardiello F, Normanno N, Maiello E, Martinelli E, Troiani T, Pisconti S, Giuliani F, Barone C, Cartenì G, Rachiglio AM, et al: Clinical activity of FOLFIRI plus cetuximab according to extended gene mutation status by next-generation sequencing: Findings from the CAPRI-GOIM trial. Ann Oncol 25: 1756-1761, 2014.
15. Ciardiello F and Normanno N: HER2 signaling and resistance to the anti-EGFR monoclonal antibody cetuximab: A further step toward personalized medicine for patients with colorectal cancer. Cancer Discov 1: 472-474, 2011.

16. Misale S, Di Nicolantonio F, Sartore-Bianchi A, Siena S and Bardelli A: Resistance to anti-EGFR therapy in colorectal cancer: From heterogeneity to convergent evolution. Cancer Discov 4: 1269-1280, 2014

17. Yang ZY, Wu XY,Huang YF, Di MY,Zheng DY, Chen JZ, Ding H, Mao C and Tang JL: Promising biomarkers for predicting the outcomes of patients with KRAS wild-type metastatic colorectal cancer treated with anti-epidermal growth factor receptor monoclonal antibodies: A systematic review with meta-analysis. Int J Cancer 133: 1914-1925, 2013.

18. Guinney J, Ferté C and Dry J, McEwen R, Manceau G, Kao KJ, Chang KM, Bendtsen C, Hudson K, Huang E, et al: Modeling RAS phenotype in colorectal cancer uncovers novel molecular traits of RAS dependency and improves prediction of response to targeted agents in patients. Clin Cancer Res 20: 265-272, 2014.

19. Montagut C, Dalmases A, Bellosillo B, Crespo M, Pairet S, Iglesias M, Salido M, Gallen M, Marsters S, Tsai SP, et al: Identification of a mutation in the extracellular domain of the epidermal growth factor receptor conferring cetuximab resistance in colorectal cancer. Nat Med 18: 221-223, 2012.

20. Arena S, Bellosillo B, Siravegna G, Martínez A, Cañadas I, Lazzari L, Ferruz N, Russo M, Misale S, González I, et al: Emergence of multiple EGFR extracellular mutations during cetuximab treatment in colorectal cancer. Clin Cancer Res 21: 2157-2166, 2015.

21. Van Emburgh BO, Arena S, Siravegna G, Lazzari L, Crisafulli G, Corti G, Mussolin B, Baldi F, Buscarino M, Bartolini A, et al: Acquired RAS or EGFR mutations and duration of response to EGFR blockade in colorectal cancer. Nat Commun 7: 13665, 2016.

22. Montagut C, Siravegna G and Bardelli A: Liquid biopsies to evaluate early therapeutic response in colorectal cancer. Ann Oncol 26: 1525-1527, 2015. 